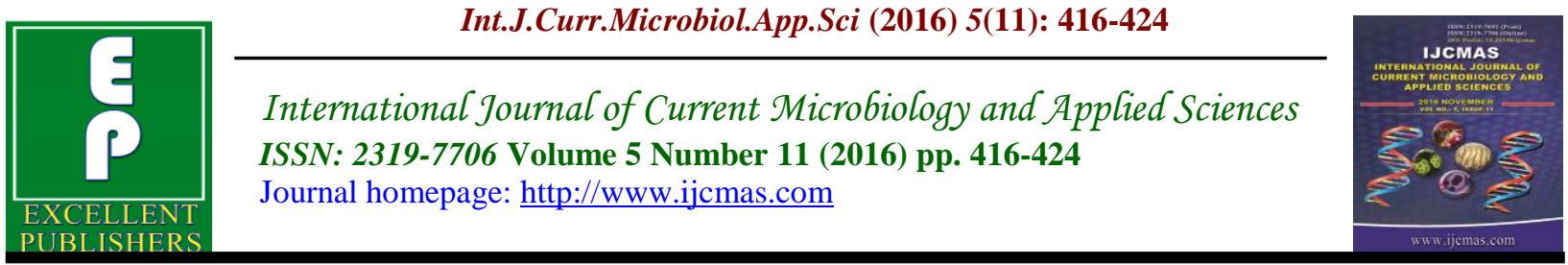

Original Research Article

http://dx.doi.org/10.20546/ijcmas.2016.511.048

\title{
Prevalence of Vancomycin Resistant Enterococcus and its Antibiotic Resistance Pattern at a Tertiary Care Hospital
}

\author{
Pradnya Atmaram Jadhav ${ }^{1^{*}}$ and Shahriar B. Roushani \\ Department of Microbiology, Bharati Vidyapeeth Deemed University Medical College and \\ Hospital, Sangli- 416414, Maharashtra, India \\ *Corresponding author
}

\section{Keywords}

Urinary tract infection, Enterococcus faecalis, Enterococcus faecium, E test, Vancomycin resistant Enterococcus, High level amino glycoside resistant Enterococcus.

\section{Article Info}

Accepted:

23 October 2016

Available Online:

10 November 2016

\section{A B S T R A C T}

UTI is one of the most common infectious diseases in people of all age groups from the neonates up to geriatric age groups and geographical locations are affected and thus represent a major source of human discomfort. Since the mid 1990's, hospital-associated Enterococcal bacteriuria, has shown a significant increase in frequency over time and is the second most frequently isolated urinary pathogen. Enterococcus faecalis is more common in nosocomial infections than E. faecium, but $E$. faecium has a great ability to acquire drug resistance. The present study was planned to determine the antimicrobial susceptibility pattern of urinary isolates of Enterococcus species and the emergence of vancomycin resistance and high level aminoglycoside resistance. A total number of 187 urinary isolates of enterococci were tested for phenotypic characteristics by conventional methods and growth on hicrome UTI agar. Antimicrobial susceptibility pattern was studied by Kirby-bauer disk diffusion technique, screening agar method and minimum inhibitory concentration (MIC) testing by $\mathrm{E}$ test to determine vancomycin resistance. Out of 187 enterococcal isolates, 149 (79.67\%) were Enterococcus faecalis and 38 (20.32\%) were Enterococcus faecium. Among predisposing factors in the present study, catheterization was found to be the most important predisposing factor where the highest number of patients $(43.89 \%)$ had enterococcal UTI, followed by diabetes accounted for $31.23 \% .13$ enterococcal isolates were resistant to vancomycin by $\mathrm{E}$ test and all the isolates were susceptible to teicoplanin, linezolid and tigecycline. Maximum resistance was observed against high level amino glycoside (HLAR), i.e.50.8\%. Followed by, most of the isolates were resistant to tetracycline and ampicillin, i.e $44.3 \%$ and $42.7 \%$ respectively. Multidrug resistant enterococci especially resistant to vancomycin and aminoglycosides have become a threat to patient's safety, making it a formidable nosocomial pathogen.

\section{Introduction}

UTI is one of the most common infectious diseases in people of all age groups from the neonates up to geriatric age groups and geographical locations are affected and thus represent a major source of human discomfort (Pankaj et al., 2012). Of the various uropathogens, the most common organisms are E.coli, Enterococcus spp, 
Staphylococcus aureus and Klebsiella spp. (Sivasankari et al., 2013).

Since the mid 1990's, hospital-associated Enterococcal bacteriuria, has shown a significant increase in frequency over time and was the second most frequently isolated urinary pathogen (Wazait et al., 2003). In a CDC survey of nosocomial infection, Enterococci accounted for $13.9 \%$ of urinary tract infections (Desai et al., 2001). This organism is considered as second leading cause of hospital acquired infections (Bose et al., 2012). Enterococcus faecalis is more common in nosocomial infections than $E$. faecium, but $E$. faecium has a great ability to acquire drug resistance (Mohammad et al., 2008).

The treatment of these infections poses a great challenge due to the inherent resistance of Enterococcus to many antibiotics. A combination of penicillin and gentamicin had been the mainstay of treatment of enterococcal infections till now but with the emergence of high level aminoglycoside resistance (HLAR), vancomycin is the only alternative available. The widespread use of glycopeptides in hospitals has led to the emergence of vancomycin resistant Enterococcus (VRE) which is a major concern for health care professionals (Mathur et al., 2003).

In this study, we attempted to determine the antimicrobial susceptibility pattern of urinary isolates of Enterococcus species and the emergence of vancomycin resistance and high level aminoglycoside resistance in those isolates recovered from the patients at a Pravara Tertiary Care Hospital, Loni, during March 2011 to August 2012.

\section{Materials and Methods}

This study was conducted after approval from institutional ethical committee, in the
Department of Microbiology, Pravara Medical College and Hospital and included a total number of 187 enterococci isolated from urine from the patients irrespective of age, sex or antibiotic therapy. Relevant information regarding the patient's history as well as signs and symptoms of patients was collected.

Presence of $>10$ pus cells/hpf in centrifuged urine and $>5$ pus cells/hpf in uncentrifuged urine is considered to be significant (Taneja et al., 2004). Culture was done on blood agar, MacConkey's agar, CLED agar (Himedia M792) and Hicrome UTI agar (Modified M1418) and plates were incubated aerobically at $37^{\circ} \mathrm{C}$ for 24 hours (Bose et al., 2012).

Enterococcus showed greenish yellow colour on CLED agar and bluish green on Hicrome UTI agar. All of the enterococcal isolates were tested for phenotypic characteristics by conventional methods, on the basis of the following criteria: growth on bile esculin agar and in $6.5 \% \mathrm{NaCl}$ broth, absence of catalase, and presence of pyrolidonyl arylamidase. Species-level identification was performed by biochemical tests including acid fermentation of mannitol, sorbitol, sucrose, arabinose, and raffinose; motility; and arginine hydrolysis (Mohammad et al., 2008).

For studying the antimicrobial susceptibility pattern in enterococcal isolates, three methods were used Kirby-bauer disk diffusion technique, screening agar method and minimum inhibitory concentration (MIC) testing by $\mathrm{E}$ test to determine vancomycin resistance.

Kirby bauer disk diffusion method was used for determining the susceptibility of the isolates to the commonly used antibiotics against Enterococcus spp. using the standard guidelines issued by the Clinical 
Laboratories Standards Institute (CLSI). The antibiotics tested were nitrofurantoin (300 $\mu \mathrm{g})$, ampicillin $(10 \mu \mathrm{g})$, ciprofloxacin (5 $\mu \mathrm{g})$,gentamicin (high content) $(120 \mu \mathrm{g})$, tetracycline $(30 \mu \mathrm{g})$, vancomycin $(30 \mu \mathrm{g})$, teicoplanin $(30 \mu \mathrm{g})$, linezolid $(30 \mu \mathrm{g})$ and tigecycline $(15 \mu \mathrm{g})$. All antibiotic discs were obtained from Hi Media Pvt Ltd, India.

Screening for low level vancomycin resistance for the isolates resistant to vancomycin by disk diffusion method was done by vancomycin screen agar method using $6 \mu \mathrm{g} / \mathrm{ml}$ vancomycin according to NCCLS recommendations. Minimum inhibitory concentration (MIC) of vancomycin was performed by $\mathrm{E}$ test $(\mathrm{AB}$ Biodisk, Solna, Sweden) (Mendiratta et al., 2008). Susceptibility interpretations followed the guidelines proposed by CLSI ( $\mathrm{S} \leq 4 ; \mathrm{I}=8 / 16 ; \mathrm{R} \geq 32 \mathrm{mg} / \mathrm{L})$ (Manpreet et al., 2014). Enterococcus faecalis (E. faecalis) ATCC 29212 and Staphylococcus aureus ATCC 25923 were used as control strains (Bose et al., 2012).

\section{Results and Discussion}

Total 187 enterococci were isolated from urine specimens over a period of one and half year. Number of females infected with enterococcal infection was more, i.e. 122 $(65.24 \%)$ and the number of males affected was 65 (34.75\%). Male: Female ratio was $1: 1.87$ as shown in figure 1 .

Maximum number of patients affected belongs to $21-30$ years of age group, followed by $31-40$ years. The age group, 0 - 10 years, has minimum number of patients. (figure 2)

Among 187 enterococcal isolates, 149 (79.67\%) were Enterococcus faecalis (E. faecalis) and $38 \quad(20.32 \%)$ were Enterococcus faecium (E. faecium). (Table 1)
Among predisposing factors in the present study (Table 2), catheterization was found to be the most important predisposing factor where the highest number of patients $(43.89 \%)$ had enterococcal UTI, followed by diabetes accounted for $31.23 \%$. Pregnancy was also an important predisposing factor and $24.88 \%$ of pregnant patients showed enterococcal UTI.

Out of 187 enterococcal isolates, 13 were resistant to vancomycin by $\mathrm{E}$ test and all the isolates were susceptible to teicoplanin, linezolid and tigecycline. Maximum resistance was observed against high level amino glycoside (HLAR), i.e.50.8\%. Followed by, most of the isolates were resistant to tetracycline and ampicillin, i.e $44.3 \%$ and $42.7 \%$ respectively. (Table 3 )

Recent years have witnessed increased interest in enterococci not only because of their ability to cause serious infections but also because of their increasing resistance to many antimicrobial agents (Marothi et al., 2005).

In the present study, of the 187 enterococcal isolates, $79.67 \%$ were E. faecalis while prevalence of $E$. faecium was $20.32 \%$ (Table 1), similar to the results stated by Marothi et al from Madhya Pradesh (Marothi et al., 2005). According to study from Tamilnadu and Jalgaon( Maharashtra) $78.8 \%$ and $86 \%$ were E.faecalis whereas $21.2 \%$ and $14 \%$ were E.faecium, respectively (Sivasankari et al., 2013; Bhardwaj et al., 2013). It is also stated that Enterococcus faecalis is more common in nosocomial infections than $E$. faecium (Mohammad et al., 2008).

As per the correlation of enterococcal UTI with the predisposing factors in our study (Table 2), catheterization was found to be the most important one i.e $43.89 \%$, followed by diabetes $(31.23 \%)$ and pregnancy 
accounted for $24.88 \%$. Richard Difuku et $a l$. , and Robert Orenstein et al., reported the catheter associated UTI constitute $35-40 \%$ of all UTIs (Richard Difuku et al., 1984, Robert Orenstein et al., 1999). Closely similar to our result, Patil et al in 2012 noted that diabetes found in $36 \%$ of all UTIs (Patil et al., 2012). However, studies from Chennai and Iran have reported the incidence of UTI in type 2 diabetic patients was as high as 42-42.8\% (Janifer et al., 2009, Richard Daifuku et al., 1984). Changes in the host defence mechanism, the prevalence of diabetic cystopathy and of microvascular disease in the kidneys may play a role in higher incidence of UTI in diabetic patients (Janifer et al., 2009).

The drawback of the control and treatment of enterococcal infections is their intrinsic resistance to various antibiotics, their capabilities to develop new resistance and to live in the external environment for a longer time (Gülçin Baldýr et al., 2013). The emergence and spread of glycopeptide resistance in enterococci has become a substantial clinical and epidemiological concern, (Ojan Assadian et al., 2007).

Vancomycin Resistant Enterococci (VRE) takes place among the important nosocomial pathogens, in that the treatment options are limited, it is likely to transfer vancomycin resistance to other pathogens. VRE is known to spread in the hospital setting through contaminated hands and surfaces (Gülçin Baldýr et al., 2013).

In the present study, from 187 enterococcal isolates, $13(6.9 \%)$ were resistant to vancomycin but susceptible to teicoplanin, similar to the result studied by Hajia et al., (Hajia et al., 2012). The Studies from Puducherry and Chandigarh have reported $8.7 \%$ and $5.5 \%$ incidence of VRE, respectively (Praharaj et al., 2013). Although the prevalence of VRE infections in India is much lower than in the western world, it has been increasing in the past one decade. (Praharaj et al., 2013). Antibiotic selective pressure exerted by extensive use of third generation cephalosporins and drugs with potent activity against anaerobes have been reported to predispose to VRE colonization and infection (Taneja et al., 2004).

The VRE strains containing vanA and vanB genes carry a high level of resistance to vancomycin, while those carrying VanC gene show a low level of resistance to vancomycin. The strains with vanA gene are resistant to teicoplanin as well as vancomycin (Gülçin Baldýr et al., 2013). And VanB type resistance is characterized by resistance to vancomycin and susceptibility to other glycopeptides like teicoplanin since only the former antibiotic is capable of inducing the vanB resistance type (Guido Werner et al., 2012). So, based on the results of the MIC studies and susceptibility to teicoplanin, in our study, it appears that Van B may be the phenotype for vancomycin resistant E. faecalis and $E$. faecium (Taneja et al., 2004; Gülçin Baldýr et al., 2013).

The emergence of VRE had seriously affected the treatment of the conditions caused by this organism. This leaves clinicians a limited choice. For these types of cases, newer antibiotics, such as linezolid and tigecycline are useful. Linezolid was the first oxazolidinone to be available for clinical use in 2000. It has activity against both E. faecium and E. faecalis (Praharaj et $a l ., 2013)$. It binds to the domain $\mathrm{V}$ region of $23 \mathrm{~S}$ rRNA and mutation to that domain causes resistance to the drug (Bose et al., 2012). Another advantage of this drug is that it can be administered both intravenously and orally (Praharaj et al., 2013). Outbreaks due to linezolid resistant enterococci, though rare, have been reported recently. Also the 
cases of linezolid- resistant vancomycinresistant $E$. faecium infection without any prior exposure to linezolid have been reported (Praharaj et al., 2013). Tigecycline is a new glycylcycline derivative of tetracycline. Tissue penetration of tigecycline is excellent and it acts against both Gram positive and Gram negative microorganisms (Bose et al., 2012). All the VRE isolates in our study were found to be sensitive to linezolid and tigecycline.

Gentamicin is one of the most commonly used aminoglycosides against enterococci (Mohammad et al., 2008). The cell wall inhibitors such as penicillin, ampicillin, or vancomycin have been administered in combination with the aminoglycosides such as streptomycin and gentamicin in the treatment of serious infections caused by enterococci. A synergistic effect between the cell wall synthesis inhibitors and aminoglycosides disappears in the presence of high-level resistance to aminoglycoside and causes difficulties in the treatment of severe enterococcal infections (Gülçin Baldýr et al., 2013).

Enterococci have intrinsic low-level resistance to aminoglycosides and, in addition, have acquired aminoglycoside resistance genes. Along with the rapid rise in incidence of resistance in enterococci to traditional antibiotics, including aminoglycosides, transmission of multiresistant and HLGR strains has been reported (Mohammad et al., 2008).

High level gentamicin resistance (HLGR) was first time reported in E. faecalis in the year 1979. Resistance to amino glycoside is often associated with multidrug resistance and is due to various amino glycoside modifying enzymes. Moreover, E.faecium has become difficult to be treated by glycopeptides and amino glycosides (Bose et al., 2012).
In our study, 95 (50.8\%) Enterococcal isolates were resistant to Gentamicin (high level). In another study, from the same place, high level gentamicin resistance was found to be 58\% (Bose et al., 2012). A study by MATHUR et al from Northen India has stated $26 \%$ high level gentamicin resistance. (Mathur et al., 2003) and Praharaj et al found $37 \%$ of all Enterococcus isolates were high-level gentamicin resistant by disk diffusion method (Praharaj et al., 2013).

Recently, Chandrakanth et al., (2012); Shafiyabi et al., (2013), and Jada and Kumar (2013) also reported Enterococci to exhibit resistance to Gentamicin. On the other hand, Acharya et al., (2003) and Sreeja et al., (2012) found the isolates to be $62 \%$ and $55.2 \%$ susceptible to this antibiotic respectively (Manpreet Kour et al.,2014). The presence of HLGR is predictive of the loss of synergy between gentamicin and a cell wall-active agent such as ampicillin or vancomycin (Sivasankar et al., 2013).

Ampicillin resistance in enterococci may be caused either by change in penicillinbinding proteins or rarely by production of a beta-lactamase enzyme (Gülçin Baldýr et al., 2013). In the present study, $42.7 \%$ enterococcal isolates were resistant to ampicillin. It is related with the one of the studies which shows ampicillin resistance 46.33\% (Praharaj et al., 2013). Also a study from Jaipur (Rajsthan) reported $47.16 \%$ of Enterococcal isolates were resistance to ampicillin (Manpreet Kour et al., 2014). However some of the studies have noted very low resistance to ampicillin as $12.5 \%$, $18.6 \%$ and 28.8\%.(Edet Udo et al., 2003; Gülçin Baldýr et al., 2013). And on the other hand, Mathur et al., reported $66 \%$ enterococcal strains were resistant to ampicillin (Mathur et al., 2003). 
Nitrofurantoin is an excellent drug against enterococcal urinary tract infection. It has been used for past many years and still shows very little resistance. It is both bacteriostatic and bactericidal and resistant mutants are very rare. There are no cross resistance between nitrofurantoin and other antibiotics. It is effective against both $E$. faecalis and E. faecium including most of the VRE (Bose et al., 2012).

In the present study also, $92.6 \%$ of all the enterococcal isolates were sensitive to nitrofurantoin. A study by Bose et al have reported only a $2.07 \%$ resistance to nitrofurantoin (Bose et al., 2012). Although nitrofurantoin is a good choice of drug for
UIT treatment, a study from Puducherry have reported 29\% of urinary isolates of enterococci showed in vitro resistance to it (Praharaj et al., 2013).

Again in our study 18 enterococcal isolates (9.6\%) were resistant to ciprofloxacin and $44.3 \%$ showed resistant to tetracycline. It is quite low resistant prevalence as compared to the studies by Praharaj et al., (Praharaj et al., 2013) and Mathur et al., (Mathur et al., 2003). Also a study from Puducherry showed resistance to tetracycline as high as 71.38\% (Praharaj et al., 2013) which is somewhat related with the study from Kuwait (Edet Udo et al., 2003).

Table.1 Species distribution of Enterococcal isolates from urine specimens $(\mathrm{n}=187)$

\begin{tabular}{|c|c|c|}
\hline Enterococcal species & Number & Percentage (\%) \\
\hline E. faecalis & 149 & 79.67 \\
\hline E. faecium & 38 & 20.32 \\
\hline
\end{tabular}

Table.2 Distribution of predisposing factors in patients with Enterococcal UTI $(\mathrm{n}=187)$

\begin{tabular}{|c|c|c|c|}
\hline Sr.No. & Predisposing factor & $\begin{array}{c}\text { No. of Enterococcal } \\
\text { isolates }\end{array}$ & Percentage (\%) \\
\hline 1 & Catheterisation & 82 & 43.89 \\
\hline 2 & Diabetes & 58 & 31.23 \\
\hline 3 & Pregnancy & 47 & 24.88 \\
\hline
\end{tabular}

Table.3 Antimicrobial resistance pattern of Enterococcal isolates $(\mathrm{n}=187)$

\begin{tabular}{|c|l|c|c|}
\hline Sr.No. & Antibiotics & No. of resistant strains & \% of resistant strains \\
\hline 1 & Tetracycline & 83 & 44.3 \\
\hline 2 & Ampicillin & 80 & 42.7 \\
\hline 3 & Gentamicin(high level) & 95 & 50.8 \\
\hline 4 & Teicoplanin & 0 & 0 \\
\hline 5 & Vancomycin & 13 & 6.9 \\
\hline 6 & Linezolid & 0 & 0 \\
\hline 7 & Tigecycline & 0 & 0 \\
\hline 8 & Nitrofurantoin & 14 & 9.4 \\
\hline 9 & Ciprofloxacin & 18 & \\
\hline
\end{tabular}


Fig.1 Sex wise distribution of patients with Enterococcal UTI ( $\mathrm{n}=187)$

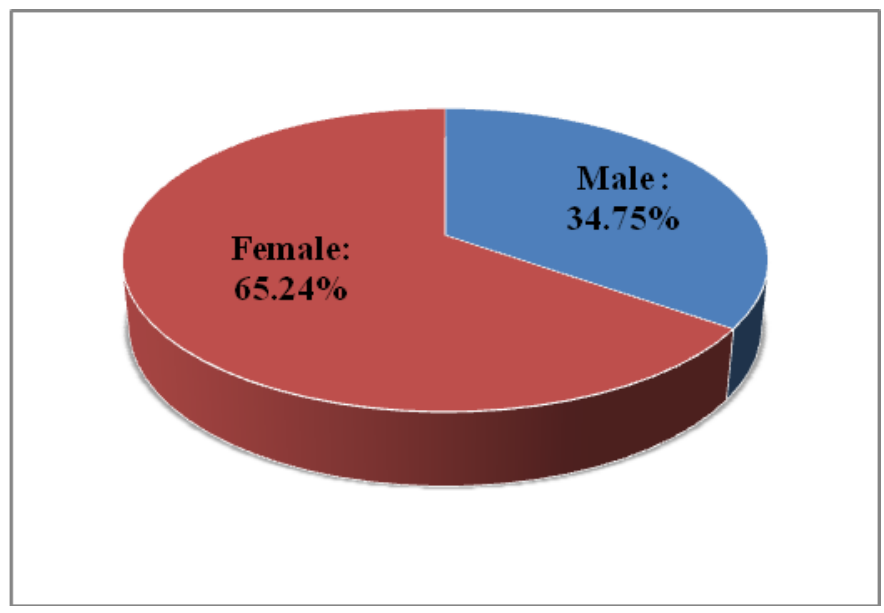

Fig.2 Age group wise distribution of all patients $(\mathrm{n}=187)$

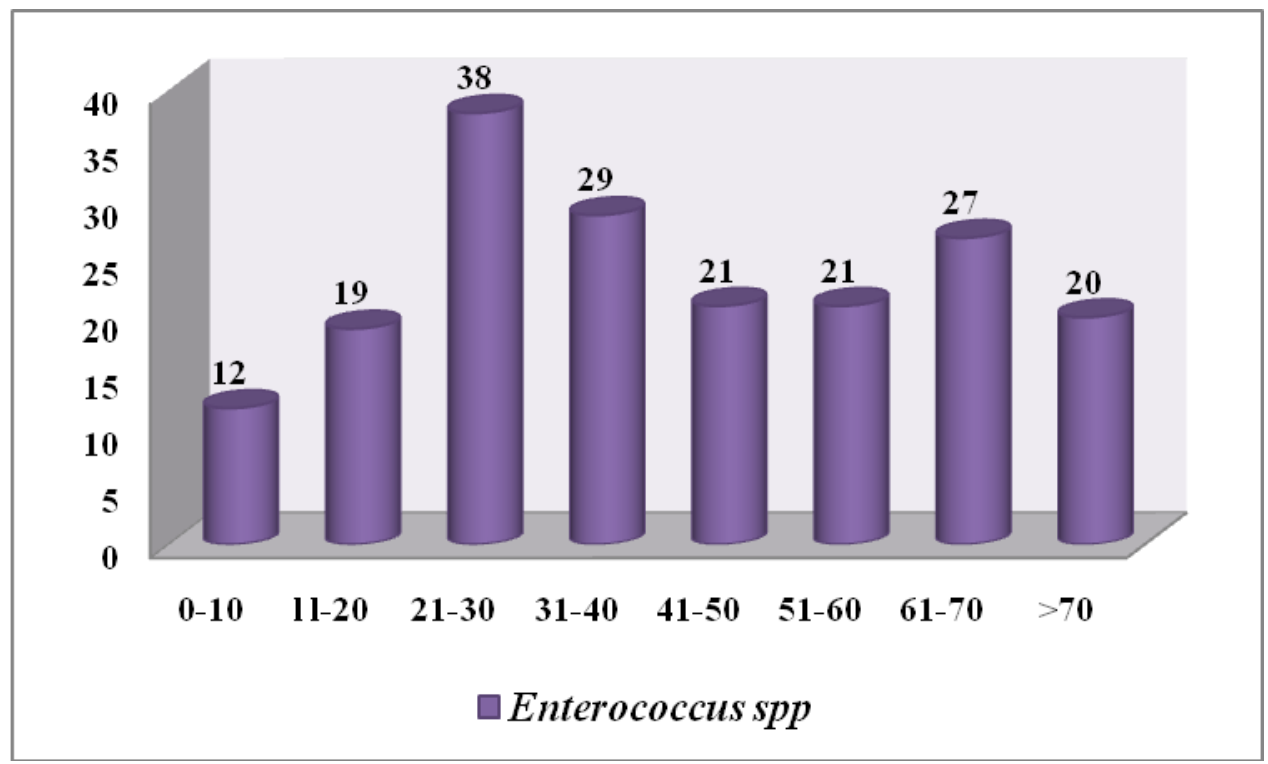

In conclusion, multidrug resistant enterococci especially resistant to vancomycin and aminoglycosides have become a threat to patient's safety, making it a formidable nosocomial pathogen. The rising prevalence of antimicrobial resistance trait among Enterococcus spp. has critical outcome on health care system due to increasing in mortality as a result of existence of severe infections such as endocarditis without any effective antimicrobial therapeutic agents. This fact highlights the importance of strict enforcement of antibiotic policies coupled with greater adherence to infection control measures to prevent emergence and spread of antibiotic resistant bacteria.

\section{References}

Desai, P.J., D. Pandit, M. Mathur, A. Gogate. 2001. Prevalence, identification and distribution of various species of Enterococci isolated 
from clinical specimens with special reference to UTI in Catheterized patients. Ind. J. Med. Microbiol., 19(3): 132-137,

Edet, E., Udo, Noura Al-Sweih, Oludotun A. Phillips, Tulsi, D., Chugh. 2003. Species prevalence and antibacterial resistance of enterococci isolated in Kuwait hospitals. J. Med. Microbiol., 52, 163-168.

Eshwarappa, M., $\quad$ R. Dosegowda, I. Vrithmani Aprameya, M.W. Khan, P. Shiva Kumar, P. Kempegowda. 2011. Clinico-microbiological profile of urinary tract infection in South India. Indian J. Nephrol., 21(1): 30-36.

Guido Werner, Ingo Klare, Carola Fleige, Uta Geringer, Wolfgang Witte, Heinz Michael Just, Renate Ziegler. Vancomycin-resistant vanB-type Enterococcus faecium isolates expressing varying levels of vancomycin resistance and being highly prevalent among neonatal patients in a single ICU. Antimicrobial Resistance and Infection Control, 1: 21.

Gülçin Baldýr, Derya Öztürk Engin, Metin Küçükercan, Asuman Ýnan, Seniha Akçay, Seyfi Özyürek, Sebahat Aksaray. High-level resistance to aminoglycoside, vancomycin, and linezolid in enterococci strains. Baldýr G., et al., Resistance, J. Microbiol. Infectious Dis., JMID, 3(3): 100-103.

Ira Praharaj, S., Sujatha, Subhash Chandra Parija. 2013. Phenotypic \& genotypic characterization of vancomycin resistant Enterococcus isolates from clinical specimens. Indian J. Med. Res., 138, pp 549-55.

Janifer, J., S. Geethalakshmi, K. Satyavani, V. Viswanathan. 2009. Prevalence of lower urinary tract infection in South Indian type 2 diabetic subjects. Indian J. Nephrol., vol 19: Issue 3.
Manjunath, G.N., Prakash, R., Vamseedhar Annam, Kiran Shetty. 2011. Changing trends in the spectrum of antimicrobial drug resistance pattern of uropathogens isolated from hospitals and community patients with UTI in Tumkur and Bangalore. Int. J. Biol. Medical res., 2(2): 504-.507

Manpreet Kour, I., Soni, Rajni Sharma. 2104. Antimicrobial Susceptibility of Biofilm Producing Enterococcus Faecalis Isolated From Clinical Samples. Indian J. Appl. Res., Volume : 4 | Issue : $11 \mid$ November 2014 | ISSN - 2249 555X.

Marothi, Y.A., H. Agnihotri, D. Dubey. 2005. Enterococcal resistance- An Overview. Indian J. Med. Microbiol., 23(4): 214-9.

Massoud Hajia, Mohammad Rahbar, Mona Mohammad Zadeh. 2012. A novel method "CHROMagar" for screening vancomycin-resistant enterococci (VRE) isolates. African J. Biotechnol., Vol. 11(41), pp. 9865-9868.

Mendiratta, D.K., H. Kaur, V. Deotale, D.C. Thamke, R. Narang, P. Narang. 2008. Status of high level aminoglycoside resistant Enterococcus faecium and Enterococcus faecalis in a rural hospital of Central India. Indian J. Med. Microbiol., 26(4): 369-71

Mohammad Mehdi Soltan Dallal, Mahnaz Saifi, Mohammad Reza Pourshafie, Mohammad Reza Eshraghian. HighLevel Gentamicin-Resistant Enterococcal Isolates From Urinary Tract Infection in Iran. Infect. Dis. Clin. Practice, Volume 16, Number 1.

Neelam Taneja, Phulan Rani, Rekha Emmanuel, Meera Sharma. 2004. Significance of vancomycin resistant enterococci from urinary specimens at a tertiary care centre in northern India. Indian J. Med. Res., pp 72-74. 
Nilima, R., Patil, Ulhas, S., Mali, Meena, N. Ramtirthkar, Poorva, A. Bhave. (Sule), Shivdas, S., Mali, Vijay, S. Mane. 2012. Bacteriuria in diabetic patients. World J. Sci. Technol., 2(12): 25-27.

Ojan Assadian, Mehrdad Askarian, Maria Stadler, Soheila Shaghaghian. Prevalence of Vancomycin-Resistant Enterococci colonization its risk factors in chronic hemodialysis patients in Shiraz, Iran. $B M C$ Infectious Dis., 7: 52.

Pankaj Baral, Sanjiv Neupane, Bishnu Marashini, Kasha Ghimire. High prevalence of multidrug resistance in bacterial uropathogens from Kathmandu, Nepal. BMC Res. Notes, 5: 38 .

Purva Mathur, Arti Kapil, Rachna Chandra, Pratibha Sharma, Bimal Das. Antimicrobial resistance in Enterococcus faecalis at a tertiary care centre of northern India. Indian $J$. Med. Res., pp 25-28.

Richard Daifuku, Walter, E., Stamm. 1984. Association of rectal and urethral colonization with urinary tract infection in patients with indwelling catheters. JAMA, vol 252: No 15.

Robert Orenstein, Edward, S., Wong. 1999. Urinary Tract Infections in Adults.
Am. Fam. Physician, 59(5): 12251234.

Seema Bose, Atindra Krishna Ghosh, Rekha Barapatre. Prevalence Of Drug Resistance Among Enterococcus Spp Isolated From A Tertiary Care Hospital. Int. J. Med. Health Sci., VolIssue-3: p 38-44.

Sivasankari, S., Somasunder, V.M., Senthamarai, S., Anitha, C., Kumudhavathi, M.S., Suneel Kumar Reddy, A., Amshavathani, S.K., Venugopal. 2013. Detection of High Level Aminoglycosides Resistant Entrococci In A Tertiary Care Hospital. IOSR J. Pharmacy and Biol. Sci., 5, PP 53-57.

Suddhanshu Bhardwaj, Kalyani Bhamre Jayashri Dhawale, Mahendra Patil, Sunil Divase. Enterococcus faecium and Enterococcus faecalis, the nosocomial pathogens with special reference to multi-drug resistance and phenotypic characterization. Int. J. Pharma. Sci. Practice, Volume 2, Number 1, pp 1-10.

Wazait, H.D. 2003. Catheter-associated urinary tract infections: prevalence of uropathogens and pattern of antimicrobial resistance in a UK hospital (1996-2001). BJU Int., 91(9): p. 806-809.

\section{How to cite this article:}

Pradnya Atmaram Jadhav and Shahriar B. Roushani. 2016. Prevalence of Vancomycin Resistant Enterococcus and its Antibiotic Resistance Pattern at a Tertiary Care Hospital. Int.J.Curr.Microbiol.App.Sci. 5(11): 416-424. doi: http://dx.doi.org/10.20546/ijcmas.2016.511.048 